\title{
33. Ein Haus - zwei Brüder: \\ Die Verleger Hans Dieter und Wolfgang Beck
}

A m 27. September 1968 schrieben neun Lektoren des Suhrkamp-Verlags einen Brief an

Verleih uns Frieden gnädiglich. Martin Luther

Siegfried Unseld und verlangten die Umsetzung einer Lektoratsverfassung, mit der man eine Sozialisierung der deutschen Verlage einleiten wollte. Programm- und Personalentscheidungen sollten künftig auf einer Versammlung, der alle Lektoren angehörten, mit einfacher Mehrheit entschieden werden. Unseld war ob dieses «heimtückischen Anschlags» höchst beunruhigt und arbeitete einen Notfallplan aus, der die Entmachtung seiner Lektoren vorsah, die vorübergehend durch Suhrkamp-Autoren, darunter Jürgen Habermas und Martin Walser, ersetzt werden sollten. Einen guten Monat später war der Spuk vorüber: Einige linke Lektoren gingen, der Rest arrangierte sich mit der neuen Geschäftsordnung, die dem Unternehmer das letzte Wort garantierte. ${ }^{1}$ Eine solche Palastrevolution hat es bei C.H.Beck nie gegeben. Doch kam es auch in diesem Unternehmen zu zeitbedingten Veränderungen.

\section{Mehr Demokratie wagen}

Als Wolfgang Beck 1973 in den Verlag eintrat, setzte er den patriarchalischen Führungsstil seines Vaters nicht fort. ${ }^{2}$ Gewiss, starre Strukturen und autoritäres Gebaren waren schon Heinrich Beck fremd gewesen. Aber er war der Seniorchef, der das letzte Wort hatte. Sein Sohn konnte und wollte mit $3_{1}$ Jahren nicht alle Vorgänge kontrollieren und war sich wohl bewusst, dass er der Unterstützung im Verlagsgeschäft bedurfte. Rat und Hilfe nahm er bereitwillig an, und er nutzte die kurzen Wege der Kommunikation im Haus. Der Verleger Wolfgang Beck und sein Cheflektor ErnstPeter Wieckenberg bildeten gemeinsam mit Jürgen Fischer in der Herstellungsleitung und, seit 1977, Eva von Freeden in der Presseabteilung das legendäre «Kleeblatt», das den Verlag in diesen Jahren entscheidend nach vorn brachte. Eine gewichtige Stimme besaß auch Günther Schiwy als langjähriger einflussreicher Lektor, der unter anderem die «Beck’sche (Schwar- 
ze) Reihe» betreute. Man rang um Entscheidungen, suchte nach Kompromissen, pflegte einen diskursiven Stil. Mitbestimmung kehrte in die Konferenzen ein. Wolfgang Beck wollte keine hierarchische Ordnung durchsetzen, sondern zur Partizipation ermutigen. Dies öffnete den Mitarbeitern neue Möglichkeiten der Entfaltung, die aber durch die Interaktion in den Konferenzen der gemeinsamen Kontrolle unterlagen.

Es war eine Zeit des Aufbruchs. In der Politik wie im Verlag. Brain-storming war an der Tagesordnung. Anregungen für Autoren und Bücher waren zahlreich. In den seltensten Fällen wurde nach einem Vorschlag sofort eine Entscheidung getroffen. Vertiefende Gespräche schlossen sich an, externe Meinungen wurden eingeholt. Aushandlungsprozesse wurden institutionalisiert und Entscheidungsprozesse liberalisiert. Zum ersten Mal in der Verlagsgeschichte wurde creative publishing durch ein Kollektiv verwirklicht.

Der «Machtwechsel» an der Verlagsspitze führte aber nicht zu einer Revision der bisherigen Programmpolitik. Das Konzept war bereits in den Killy-Konferenzen entwickelt worden: Historiker und andere Geisteswissenschaftler sollten weiter möglichst anschaulich, aber auf hohem Niveau für ein allgemeines Publikum schreiben, ohne dabei die Standards ihres Faches preiszugeben. So wurde der Verlag immer mehr zum «kulturwissenschaftlichen Publikumsverlag». Wer der Enge der fachwissenschaftlichen Produktion entkommen und eine größere Leserschaft erreichen wollte, der publizierte nun nicht mehr in akademischen Fachverlagen wie Oldenbourg, de Gruyter oder Kohlhammer, sondern suchte Häuser wie C.H.Beck auf. Zur Programmphilosophie des Hauses wurde es, Wissenschaft und Öffentlichkeit zusammenzubringen, indem sich beide ein Stück weit aufeinander zubewegten: Der Autor sollte beim Schreiben nie seinen Adressaten - ein allgemeines Publikum ohne Fachkenntnisse, aber mit Fachinteresse - aus den Augen verlieren, während das Publikum sich nicht nur trivial unterhalten lassen, sondern intellektuelle Neugierde mitbringen sollte. Beide durch entsprechende Programm- und Lektoratsarbeit zu dieser Begegnung gleichsam anzustiften wurde zum Kerngeschäft und kulturellen Auftrag des Verlags.

Das zeitgemäße Programm einer attraktiven Vermittlung von Wissen durch neue Formate und das ebenfalls zeitgemäße Modell einer Demokratisierung der Entscheidungsprozesse durch die Einführung flacher Hierarchien im Verlag waren höchst erfolgreich. Wolfgang Beck hatte folglich keine Veranlassung, seinen Führungsstil zu ändern. Dennoch gab es im Laufe der Jahrzehnte deutliche Zäsuren. Von nicht zu unterschätzender 
Bedeutung war der Aufbau einer eigenen Presseabteilung in der zweiten Hälfte der siebziger Jahre. Die Aufgabe meisterte auf höchst eindrucksvolle Weise Eva von Freeden. Die Tochter eines Kunsthistorikers hatte eine Buchhandelslehre gemacht und später im Würzburger Arena Verlag die Presse- und Lizenzabteilung eingerichtet. Ihren Job bei C.H.Beck trat sie am 15. Februar 1977 an. Sie war Wolfgang Becks erste Akquisition. Während belletristische Verlage schon länger ihre Öffentlichkeitsarbeit professionalisiert hatten, hinkten wissenschaftliche Verlage immer noch hinterher. Die Verleger reisten, besuchten Buchhandlungen, führten Gespräche und erzählten von ihrem Programm und ihren Autoren. Als Eva von Freeden zu C.H.Beck kam, gab es zwar eine Werbeabteilung; für den Kontakt zur Presse waren aber in der Regel die Lektoren zuständig. Eva von Freeden hat diese überkommenen Strukturen revolutioniert. Ihre Anfänge waren nicht einfach. Sie musste einen eigenen Etat durchsetzen, um Freiexemplare zur großzügigen Versendung an Rezensenten und Multiplikatoren - Persönlichkeiten, von denen man erwarten durfte, dass sie etwas für die Verbreitung eines Buches tun würden, wenn sie ein Exemplar vom Verlag erhielten - kämpfen, vor allem aber musste sie die Kontakte zu Presse, Funk und Fernsehen herstellen. Eva von Freeden kümmerte sich um Buchpräsentationen und Lesungen, Interviews und Messeauftritte; das ständig wachsende inländische Lizenzgeschäft (vor allem Vorabdrucke und auszugsweise Veröffentlichungen von Texten aus Beck-Büchern) gehörte ebenfalls zu ihrem Arbeitsbereich. Sie wusste, dass ein Buch dann besonders gut an die Medien zu vermitteln war, wenn man nicht nur über den Inhalt, sondern auch über den Autor zuverlässig zu informieren verstand. Eva von Freeden entwickelte ein dichtes Netzwerk aus Presse- und Autorenkontakten und hatte durch Letztere auch punktuell gestaltenden Einfluss auf die Programmarbeit des Hauses.

Im Gegensatz zu anderen Verlagen, in denen die Pressearbeit dem Werbe- und Vertriebsleiter unterstand, baute Eva von Freeden bei C.H.Beck eine eigene Abteilung für Öffentlichkeitsarbeit, Presse und Lizenzen auf, die bis heute direkt dem Verleger untersteht. Wahrscheinlich lag in dieser organisatorischen Selbständigkeit ein wesentlicher Grund für ihren durchschlagenden Erfolg. Bücher verschickte die Abteilungsleiterin nicht nach dem Gießkannenprinzip, getragen von der Hoffnung, dass sie in den Redaktionen schon den richtigen Rezensenten fänden. Vielmehr kannte sie sehr genau ihre je nach Genre und Titel unterschiedlich geeigneten Ansprechpartner in den Redaktionsräumen von Presse, Funk und Fernsehen - Kontakte, die sie ebenso professionell wie liebevoll pflegte. Ihre 
Personalkenntnisse und guten Verbindungen halfen, dem Haus die mediale Präsenz zu verschaffen, die es benötigte, um die Idee des wissenschaftlichen Publikumsverlags realisieren zu können. Wie souverän «die Freeden», wie sie in der Branche bald mit Respekt genannt wurde, ihr Metier beherrschte, mag man daran ermessen, dass bald auch andere ihre Unterstützung suchten: Als Albrecht Schöne 1985 den ersten Internationalen Germanistentag in Göttingen veranstaltete, konnte er Wolfgang Beck überreden, seine Pressechefin freizustellen, damit sie das Ereignis organisierte. Als Eva von Freeden, deren Stärken Kompetenz und Diskretion waren, im Jahr 2001 ihren Abschied aus dem Verlagsgeschäft nahm, ging, wie in der «Frankfurter Allgemeinen Zeitung» zu lesen war, eine Ära zu Ende. ${ }^{3}$ C.H.Beck hinterließ sie eine zukunftstaugliche Presseabteilung, in der die alten Tugenden weiter gepflegt, aber auch neue entwickelt wurden, um den gestiegenen Herausforderungen einer zunehmend durch die Medien gesteuerten Welt gerecht zu werden.

Dies ist umso wichtiger, als Bücher heute ohne entsprechenden $\mathrm{Zu}$ spruch aus den Medien kaum noch die Wahrnehmung des Endkunden erreichen, die für die wirtschaftlich erforderlichen Absatzzahlen sorgt. Was sich nicht rasch und gut verkauft, wird vom Handel bald an die Verlage remittiert - Entwicklungszeit wird Büchern nicht mehr gegeben. Das ist der Fluch der etwa go ooo Neuerscheinungen jährlich allein auf dem deutschen Buchmarkt. Ältere Titel - die sogenannte backlist - haben nur dann eine Chance, wenn sie sich als zuverlässig verkäufliche Ware im Bewusstsein der Buchhändler eingeprägt haben und das Warenwirtschaftssystem die entsprechenden Kennzahlen auswirft. Echtes Interesse aber finden nur die aktuellen Neuerscheinungen - die sogenannte frontlist. Es ist vor diesem Hintergrund unschwer zu ermessen, weshalb die Pressearbeit über das Wohl und Wehe eines Titels, eines ganzen Programms, ja letztlich eines ganzen Verlags entscheiden kann. Von Freedens Nachfolgerin, Ulrike Wegner, führt mittlerweile vier festangestellte Vollzeit-Mitarbeiterinnen sowie einige Teilzeitkräfte und nach Bedarf eine Reihe von Volontären. Die Pressearbeit - in der es wie in kaum einem anderen Bereich auf Takt und Diplomatie ankommt - war und ist eine Domäne der Frauen.

Die Pressearbeit von C.H.Beck ist weiterhin so erfolgreich, dass der Verlag seine Stellung als einer der in den Leitmedien - «FAZ», «Zeit», «Süddeutsche Zeitung», «Neue Zürcher Zeitung», «Spiegel»- meistbesprochenen Verlage Deutschlands hat bis heute behaupten können. Ein Beispiel aus der jüngeren Vergangenheit mag genügen: In den Beilagen zur Frankfurter Buchmesse 2012, die die «Frankfurter Allgemeine Zeitung», die «Süddeut- 
sche Zeitung» und die «Zeit» herausbrachten, widmeten sich neun von 74. Besprechungen kulturwissenschaftlichen Büchern des Hauses; mit zwölf Prozent der Rezensionen war C.H.Beck am erfolgreichsten von allen Verlagen; nimmt man die belletristischen Titel hinzu, kam der Münchner Verlag auf den zweiten Platz.

Die zentrale Botschaft der Presseabteilung an die Medien - und über die Medien an Buchhandel und Endkunden - lautet, dass C.H.Beck ein Publikumsverlag ist, der konsequent auf Qualität als entscheidendes Distinktionskriterium im immer unübersichtlicher werdenden Dschungel der Massenproduktion setzt. Deshalb werden die Beck'schen Titel auf Bestenlisten, die nach Qualitätskriterien erstellt werden, und die Preise, die Autoren des Hauses erhalten, unablässig über verschiedene Kanäle kommuniziert. Wenn hier erfolgreiche Arbeit geleistet wird, so wirkt sich das auf die Attraktivität des gesamten Programms aus. Doch auch ein Bestseller kurbelt den Verkauf an: Als das Buch «Unser Jahrhundert» von Fritz Stern und Helmut Schmidt vom Erscheinungstermin im Februar 2010 bis Oktober desselben Jahres kontinuierlich auf der Bestsellerliste des «Spiegels» stand - und dort meist sogar unter den ersten zehn war -, kam dies dem Verlagsprogramm in toto zugute.

Der Verlag profitierte in den achtziger und neunziger Jahren von dem Umstand, dass die Leistungsträger in Herstellung, Presse und Lektorat nicht nur kooperierten, sondern strategisch dachten. Fachliche Kompetenz, persönliche Loyalität und unternehmerische Vision charakterisierten die Akteure. Sie waren Mitglieder eines Teams, das darauf hinarbeitete, aus C.H.Beck den bedeutendsten kulturwissenschaftlichen Verlag der Republik zu machen. Der Verleger wusste um ihre Bedeutung für C.H.Beck. Ernst-Peter Wieckenberg hat er in einer in der Verlagsbranche unüblichen Weise ganz besonders geehrt: $\mathrm{Zu}$ dessen 6o. Geburtstag veranstaltete er am 25. März 1995 im Künstlerhaus in München ein Kolloquium, dem ein gemeinsames Abendessen folgte. Mit Ute Frevert, Hans-Ulrich Wehler, Peter Gay, Arnold Esch, Hans Belting, Gustav Seibt, Ulrich Haarmann, Albrecht Schöne sowie Alexander und Barbara Demandt waren herausragende Verlagsautoren eingeladen, die zu Ehren des Cheflektors Vorträge über die «Rolle der Kulturwissenschaften in der Öffentlichkeit» hielten, über ein Thema mithin, das Ernst-Peter Wieckenberg am Herzen lag. Damit identifizierten sie sich mit seinem Lebenswerk: die deutsche Kulturgeschichtsschreibung in aufklärerischer Absicht wiederzubeleben. Sie selbst hatten durch ihre Bücher zum Erfolg dieses Projekts beigetragen. Sie schätzten wie die anderen Verlagsautoren Wieckenbergs Understatement: «Der Lek- 
tor ist nun einmal dazu bestimmt, eine dienende Rolle einzunehmen. Die mag er dann umdrehen, wenn er selber veröffentlicht, aber er sollte als Lektor allenfalls bescheiden in Erscheinung treten.» ${ }^{4}$ Sie profitierten von seinem - wie er selbst es formulierte - «altmodischen» Berufsverständnis, dass der Lektor Manuskripte noch selbst redigierte. ${ }^{5}$ Und sie respektierten ihn, weil er durch wissenschaftliche Kompetenz und bürgerliche Bildung herausragte. In seiner knapp bemessenen Freizeit publizierte er eigene Forschungserträge über Philipp Moritz, Grimmelshausen, Moses Mendelssohn, Lichtenberg, Koeppen und Dürrenmatt, um nur diese zu nennen.

Im Jahr 2000 erschien schließlich der letzte Band der «Bibliothek des 18. Jahrhunderts»: Autoren, mit denen sich Ernst-Peter Wieckenberg besonders verbunden fühlte, hatten ihre Lieblingstexte aus ebendiesem Jahrhundert zusammengetragen. Der 6 oo Seiten starke, schön gestaltete Band war eine Abschiedsgabe an den Cheflektor, der von der Planung dieses Werkes nichts wusste und mit Beginn des neuen Millenniums in den Ruhestand getreten war. 33 Jahre lang hatte er seinem Verlag als Lektor gedient.

Wer sollte Ernst-Peter Wieckenberg nachfolgen? Wolfgang Beck entschied sich für eine Lösung innerhalb des Hauses. Detlef Felken, der seit 1991 als Lektor die Neuere und Neueste Geschichte betreute, hatte sich beworben und wurde ausgewählt. Der Historiker, der in Münster und Bonn studiert hatte, kannte C.H.Beck seit seiner Arbeit im Spengler-Archiv, hatte dann während einer Mutterschaftsvertretung Ende der achtziger Jahre Verlagsluft geschnuppert und war damals Ernst-Peter Wieckenberg positiv aufgefallen. Wissenschaftlich ausgewiesen durch sein Buch über Oswald Spengler, das aus seiner Magisterarbeit erwachsen war, ferner durch seine Dissertation über die amerikanische Deutschlandpolitik unter John Foster Dulles und einige Aufsätze, entschied er sich gleichwohl gegen Wissenschaft als Beruf. Im Jahr 2000 trat Felken kein leichtes Erbe an. Die Entscheidung des Verlegers für ihn war eine Entscheidung für die Kontinuität. An der bewährten Konzeption des kulturwissenschaftlichen Verlags zu rütteln bestand kein Anlass. Es dauerte daher länger, bis der neue Cheflektor durch neue Akzente in der Programmarbeit aus Wieckenbergs Schatten treten und sichtbar seinen eigenen Stil entwickeln konnte. Wolfgang Beck nimmt seine Rolle als Verleger noch stärker wahr, nachdem mit Ernst-Peter Wieckenberg sein alter Vertrauter ausgeschieden ist und er es mit einem wesentlich jüngeren Cheflektor zu tun hat. Detlef Felken hat insbesondere das internationale Lizenzgeschäft ausgebaut, C.H.Beck auf dem globalen Markt positioniert und das Programm kosmopolitischer ge- 
macht. So hat er beispielsweise den Nobelpreisträger für Ökonomie Amartya Sen ins Programm des Verlags geholt oder auch Kwame Anthony Appiah, der als Professor für Philosophie in Princeton lehrt. Sowohl der Erwerb attraktiver Lizenzen aus dem Ausland wie der Verkauf eigener Übersetzungsrechte in andere Sprachen hat erheblich zugenommen; in der von Susanne Simor betreuten Abteilung werden heute um die $15^{\circ}$ Auslandslizenzverträge pro Jahr abgeschlossen.

Seit Detlef Felken sein Amt angetreten hat, wurde ein Programmschwerpunkt im Bereich der Biographien entwickelt. Jene Darstellungsform, die in den siebziger und achtziger Jahren mit einem Hautgout behaftet war, als man mit gutem Grund nicht länger die großen Geschichten großer Männer lesen wollte, gelangte unter seiner Ägide wieder zu Ehren. Er vertraute darauf, dass es gerade einem breiteren Publikum leichter falle, komplexe historische Entwicklungen zu verfolgen, wenn sie immer wieder mit dem Lebenslauf des Protagonisten verbunden würden. Doch sind die Anforderungen an die Kunst der Biographen gewachsen und werden explizit an die Autoren herangetragen: Gesellschaftliche, wirtschaftliche, mentalitätsund religionsgeschichtliche Kontexte müssen berücksichtigt und entsprechende Erkenntnisinteressen der Leser bedient werden, auch wenn die Darstellung einer Einzelpersönlichkeit das Leitmotiv des Werkes bildet. Auf diese Weise hat Felken eine alte Form der Geschichtsvermittlung renoviert und sie zielführend mit der gegenwärtigen Erinnerungskultur verknüpft, die auf Jahrestage und Jubiläen ausgerichtet ist.

Die größte Herausforderung besteht für Verleger und Cheflektor darin, eine konsensfähige Programmpolitik zu konzipieren, die die Balance hält zwischen auflagenstarken, marktgängigen, wirtschaftlich erfolgreichen Titeln mit einer inhaltlichen Halbwertszeit von mehreren Jahren und einer breiten Palette von Büchern hoher Qualität aus unterschiedlichen Themenbereichen, die zwischen Wissenschaft und Öffentlichkeit überzeugend vermitteln. Ein sinnfälliges Beispiel für Werke der letztgenannten Kategorie stellt das Buch des Leibniz-Preisträgers Jürgen Osterhammel «Die Verwandlung der Welt. Eine Geschichte des 19. Jahrhunderts» dar.

Doch nicht nur die Akteure im Verlag, auch die Abläufe haben sich im Laufe der Jahre verändert. Die Prozesse wurden zunehmend differenziert und diversifiziert. Die Zahl der Konferenzen hat in gleichem Maße zugenommen, wie sich die Medienwelt im Allgemeinen und die Welt der Bücher im Besonderen wandelt und beschleunigt. Als Stichwort mag etwa der Begriff e-publishing genügen. Es sind Entwicklungen, auf die der Verlag reagieren muss, um zukunftsfähig zu bleiben. 
Der wichtigste Konferenztermin ist jedoch nach wie vor die wöchentliche Lektoratskonferenz, in der über das Programm beraten wird. Das inhaltlich hochwertige und zugleich gut verkäufliche Buch bleibt das Maß der Dinge. Doch längst ist die konsequente Suche nach nicht nur gut, sondern sehr gut verkäuflichen Büchern - nach sogenannten Bestsellern - zur Pflichtübung geworden. Sie sind umso wichtiger, als solche Erfolgstitel helfen, besonders interessante, aber schwer verkäufliche Bücher mit kleineren Auflagen querzufinanzieren. Man kann davon ausgehen, dass von einem nach Umfang, Ausstattung und Ladenpreis durchschnittlichen Titel zwischen 4000 und 6000 Exemplare in überschaubarer Frist verkauft werden müssen, um alle Kosten einzuspielen.

Da die Aufwendungen in allen Arbeitsbereichen gestiegen sind, musste auch der Umsatz kontinuierlich erhöht werden. Zugleich wuchs mit der Einführung neuer Programm- und Themenbereiche die Zahl der Titel deutlich. Heute liegt die Zahl der Neuerscheinungen im kulturwissenschaftlichen und literarischen Verlagsbereich jährlich zwischen 180 und 220, was eine erhebliche Erhöhung seit den 1970er Jahren bedeutet. Hinzu kommen zahlreiche Neuauflagen, nicht wenige von ihnen auch inhaltlich überarbeitet; ihre Zahl beläuft sich auf jährlich 150 bis 170. Damit einher ging die Vergrößerung des Lektorats. Jede Lektorin und jeder Lektor hat zwar bestimmte Arbeitsschwerpunkte, doch wird erwartet, dass sie/er sich darüber hinaus auch für andere Themen interessiert und aktiv Autoren und andere - beispielsweise fremdsprachige - Buchprojekte akquiriert und realisiert. Der Cheflektor Detlef Felken ist - seit 2008 mit Unterstützung von Sebastian Ullrich - für Neuere und Neueste Geschichte verantwortlich, Stefanie Hölscher betreut mit Alexandra Schumacher Kunst, zudem Philosophie und modernes Leben, Stefan von der Lahr Altertumswissenschaften, Geschichte im Allgemeinen und Musik, Ulrich Nolte die Religionswissenschaften und außereuropäische Geschichte, Stefan Bollmann Naturwissenschaften und Philosophie, Raimund Bezold Germanistik, Sprachwissenschaft und Philosophie und Sebastian Ullrich Zeitgeschichte und Politik, ein Programmfeld, auf dem bis vor kurzem auch Christine Zeile überwiegend tätig war. Die Belletristik liegt in den Händen von Martin Hielscher und - jüngst - auch von Christiane Schmidt. Immer wieder finden auch interne Lektoratskonferenzen ohne den Verleger und den Cheflektor statt, auf denen Manuskripte und Projekte vorbesprochen werden, um sie entweder gleich abzulehnen oder an die Gesamtkonferenz weiterzuleiten.

Wie verteilen sich die Pflichten und Zuständigkeiten eines Lektors bei C.H.Beck, dessen Einstiegsgehalt etwa dem eines Wissenschaftlichen Mit- 
arbeiters an einer deutschen Universität entspricht? Auf die Manuskriptbetreuung entfallen etwa zo Prozent der Arbeitszeit. Die Tatsache, dass bei C.H.Beck nach wie vor kein Manuskript in den Satz geht, das nicht vorher redigiert worden ist, bildet das Kapital, mit dem C.H.Beck auch dann wuchern kann, wenn er sich um bereits renommierte Wissenschaftler als Autoren bemüht. Diese Erfahrung einer sorgfältigen Betreuung ist für viele der Grund, bei C.H.Beck zu veröffentlichen und dem Verlag die Treue zu halten. Dabei ist festzustellen, dass diese für den Autor entscheidende Leistung der Lektoren zu allermeist gar nicht während der Arbeitsstunden im Verlag selbst erbracht wird, wo Konferenzen, Projektentwicklung, Projektsteuerung, Absprachen mit Vertrieb und Presse und die Pflege der Autorenkontakte den Alltag bestimmen. In diesem Rahmen wäre die Unruhe viel zu groß, um sich mit der erforderlichen Sammlung mit dem Manuskript eines Autors auseinanderzusetzen. So wird die eigentliche Lektoratsarbeit in der «Freizeit` geleistet. Eine 37,5-Stundenwoche wäre angesichts der ökonomischen Realitäten und Notwendigkeiten im Verlagsgeschäft eine Illusion; Wochenarbeitszeiten von über 5o Stunden sind keine Seltenheit. Das kulturwissenschaftliche Lektorat ist die Abteilung mit dem höchsten Überstundenaufkommen. Es folgt die Presseabteilung. Der kulturwissenschaftliche Verlag hat - wie wohl auch andere vergleichbare Verlage - in diesem Bereich ein strukturelles Problem, das die Arbeitssoziologie als das Phänomen der entgrenzten Arbeit beschreibt. Die Bereitschaft, solche Leistungen zu erbringen, ist hoch; die Mitarbeiterinnen und Mitarbeiter begeistern sich für die Sache und motivieren sich untereinander.

Die Professionalisierung der Verlagsarbeit wird in besonderer Weise in den Kalkulations- und Marketingkonferenzen sichtbar. Im Rahmen der Kalkulationskonferenzen wird allwöchentlich über Auflagenhöhe, Ladenpreis, Format und Ausstattung eines jeden Titels entschieden. In diesen Sitzungen haben neben dem Verleger und der Herstellungsleitung nicht zuletzt der Vertriebsleiter André Brenner, der Cheflektor Detlef Felken und die Chefin der Presseabteilung, Ulrike Wegner, einflussreiche Stimmen. Für die Kalkulationen, die dort besprochen werden, ist die Doppelspitze in der Herstellung, Jörg Alt und Artdirectorin Konstanze Berner, verantwortlich. Konstanze Berner kommt insbesondere in Fragen der Umschlag- und Buchgestaltung, die Wolfgang Beck sehr am Herzen liegen, große Bedeutung zu - eine hochsensible, kräftezehrende Aufgabe mit vielfältigem Abstimmungsbedarf zwischen allen Akteuren: Verleger, Herstellungsleiter, Graphiker, dem zuständigen Lektor und - schlimmstenfalls - auch noch dem Autor. 
In der Marketingkonferenz, die der Kalkulationskonferenz vorausgeht, werden Presse-, Werbe- und Marketingaktivitäten diskutiert, die bestimmten Titeln zuteil werden sollen, mit denen sich thematisch und im Verkauf besondere Erwartungen verbinden. Vertriebsleiter und Pressechefin sowie der Werbeleiter Claus Beinhofer, aber beispielsweise auch der Internetadministrator Andreas Kurzal informieren den Verleger über ihre diesbezügliche Arbeit und diskutieren mit ihm Pläne und Budgetrahmen für Anzeigenwerbung, Presseaktivitäten, Lesungen, Buchpräsentationen und Werbemittel wie Gesamtverzeichnisse, Prospekte und Flyer. Ohne ihren Einsatz bestünde die Gefahr, dass selbst exzeptionelle Werke in einer medial zunehmend aufgeheizten Öffentlichkeit unbemerkt blieben.

In der Titelkonferenz, die eigentlich «Titelfindungskonferenz» heißen müsste, bemühen sich Verleger und Lektoren, für jedes Buch den richtigen Titel auszuwählen. Es werden Vorschläge aus den Lektoraten geprüft, ihre Publikumswirksamkeit und sachliche Richtigkeit diskutiert und schließlich Alternativen ventiliert. Die Teilnehmer der Konferenzen wissen, dass ein Titel durchaus über Erfolg und Misserfolg eines Buches entscheiden kann.

Da bei C.H.Beck die Presseabteilung nicht Teil der Marketingabteilung ist, entfalten beide weitreichende Aktivitäten, die das Ziel verfolgen, die Präsenz des Verlags und seiner Buchproduktion in den Medien und im Buchhandel zu optimieren. Der Vertrieb allein organisiert in der Regel über 200 Veranstaltungen pro Jahr, vornehmlich mit dem Handel in Deutschland, Österreich und der Schweiz, während die Presseabteilung etwa zo Buchpräsentationen für die verschiedenen Medien beisteuert. Es ist vor allen Dingen der Ausbau dieser beiden Abteilungen, der dazu beigetragen hat, dass seit 1970 das Personal des kulturwissenschaftlichen Verlagsteils auf fast $5^{\circ}$ festangestellte Mitarbeiterinnen und Mitarbeiter angewachsen ist. ${ }^{6}$ Die deutlich gestiegene Bedeutung der verkaufsorientierten Abteilungen manifestiert sich auch darin, dass die Leiter von Vertrieb und Presse heute selbstverständlich an den wöchentlichen Programmkonferenzen des Lektorats teilnehmen und ihre Meinung die Entscheidungsfindung beeinflussen kann.

In den letzten Jahren zeichnet sich die Tendenz ab, vermehrt den zu erwartenden ökonomisch erfolgreichen Titeln besondere werbliche Zuwendung zuteil werden zu lassen. Da die wirtschaftliche Bedeutung der backlist - auch nach 1945 noch für Jahrzehnte ein sicherer Umsatzbringer bei C.H.Beck - immer mehr nachlässt, muss der Umsatz vorrangig mit Novitäten erwirtschaftet werden. Finden sich unter diesen nicht ein paar starke 
Titel, die sich möglichst zo ooo-mal und mehr innerhalb eines Jahres verkaufen, so wirkt sich das bedenklich auf das Ergebnis aus, das im Rahmen einer Rückschaukonferenz den Abteilungsleitern, dem Lektorat sowie den Mitarbeiterinnen und Mitarbeitern in Presse, Werbung und Vertrieb zu Beginn eines jeden neuen Jahres mitgeteilt wird. Abgesehen von den Umsatzzahlen werden in dieser wichtigen Konferenz entscheidende Veränderungen der Buchhandelslandschaft vorgestellt und diskutiert; zu den regelmäßig wiederkehrenden Themen gehören beispielsweise besorgniserregende Konzentrationsprozesse im Handel, der Niedergang des kleinen und mittleren Buchhandels mit seinen für einen Qualitätsverlag als Multiplikatoren so besonders wichtigen kenntnisreichen und engagierten Buchhändlerinnen und Buchhändlern, der anscheinend unaufhaltbare Aufschwung des Internetbuchhandels und die sich zugunsten der großen Player im Buchhandelsgeschäft verändernden Rabatte.

Sind mithin alle in der Verlagsbranche aufgefordert, nach neuen umsatzstarken Projekten Ausschau zu halten, so stellt sich doch mancher Erfolg als erfreuliche und letztlich von niemandem in seiner Größe vorhersehbare Überraschung heraus, so beispielsweise das Werk von Adam Zamoyski «1812. Napoleons Feldzug in Russland», das sich im Jahr des Erscheinens - anlässlich des 2oo. Jahrestags des Scheiterns Napoleons - über 6o ooo-mal verkauft hat und sich lange auf der «Spiegel»-Bestsellerliste hat halten können. Dieser Band gehörte zu dem vom Verlag auf rund 2o Prozent geschätzten Anteil der Neuerscheinungen, die wirtschaftlich rentabel sind und die restlichen 8 o Prozent mittragen müssen.

Auf den Konferenzen wird offen diskutiert. Der Verleger seinerseits lässt die Diskussionen laufen, sieht sich selbst bisweilen eher als Bedenkenträger, der nicht gleich von einem Projekt begeistert ist, aber durch gute Argumente überzeugt werden kann. Er erinnert in solchen Runden an den Anspruch des Verlags auf Qualität und ist überzeugt, dass Qualität eine entscheidende Marketingstrategie ist. Der Erfolg gibt ihm recht, wie einige Zahlen illustrieren können: Unter den Platzierungen der Sachbuch-Bestenliste der «Süddeutschen Zeitung» und des «Norddeutschen Rundfunks» lag C.H.Beck zwischen 1992 und 2011 an der Spitze; es folgten mit deutlichem Abstand Suhrkamp, Hanser, S. Fischer, Siedler, Rowohlt und die Deutsche Verlags-Anstalt. Bei den Erstplatzierungen führte C.H.Beck in demselben Zeitraum ebenfalls die Liste an. ${ }^{7}$ Der Verlag ist regelmäßig auch auf anderen Bestenlisten zu finden, so denjenigen des «Südwestdeutschen Rundfunks» sowie der Zeitschriften «Literaturen», «damals» und «Bild der Wissenschaft». 
Um Entscheidungen wird im Verlag nach wie vor hart gerungen. Verlagskonferenzen sind im Luhmann'schen Sinne soziale Systeme, in denen die doppelte Kontingenz durch Kommunikation überwunden wird: «Jeder fungiert - wenn nicht zugleich, so doch im raschen Wechsel - als Handelnder und als Beobachter und gibt beide Positionen in den Kommunikationsprozess ein.» ${ }^{8}$ Nach einer kontroversen Debatte ist die Affirmation des Ergebnisses durch Verleger und Lektoren wichtig. Der Erfolg des kulturwissenschaftlichen Verlagsteils beruht nicht zum geringsten Teil auf der konsensorientierten Diskussionskultur des Hauses.

\section{Verlag und Verleger}

Der Gesamtverlag C.H.Beck schreibt bis heute seine Erfolgsgeschichte fort. Seit 1973 wuchs der Gesamtumsatz um mehr als das Achtfache auf inzwischen rund 150 Millionen Euro. ${ }^{9}$ Die Lokomotive des Verlags ist, wie schon in den Zeiten zuvor, der juristische Zweig. Der kulturwissenschaftliche Verlagsteil expandierte im gleichen Zeitraum ebenfalls erheblich, weist im langjährigen Durchschnitt aber nur rund zehn Prozent des Gesamtumsatzes aus. Doch dies ist lediglich eine Form der Bilanzierung. Denn ein Verlag lebt immer auch von dem symbolischen Kapital, das er erwirtschaftet. So wie der juristische Verlagsteil in seiner Wirtschaftsleistung den kulturwissenschaftlichen deutlich überragt, so überragend ist der Beitrag des kulturwissenschaftlichen Verlagsteils zum intellektuellen Profil des Hauses in der Öffentlichkeit. Völlig zu Recht wurde Wolfgang Beck in seltener Einstimmigkeit der Jury zum Verleger des Jahres 2011 gewählt. ${ }^{10}$

Die wirtschaftliche Prosperität des Unternehmens zeigt sich auch in Zukäufen und Neugründungen. Der juristische Fachverlag hat nach der Öffnung des osteuropäischen Marktes Niederlassungen in Polen, Rumänien, Tschechien und Russland gegründet. Nur die Zweigstelle in Moskau erwies sich wegen der mafiösen Strukturen vor Ort als nicht lebensfähig. Sie wurde wieder geschlossen. Der Schweizer Verlag Helbing \& Lichtenhahn, der ebenfalls auf Recht, Steuern und Wirtschaft spezialisiert ist, wurde 1998 erworben. 1999 kaufte C.H.Beck von Springer den juristischen und sozialwissenschaftlichen Fachverlag Nomos in Baden-Baden. Vier Jahre später inkorporierte man den Kommunal- und Schul-Verlag in Wiesbaden. 2005 wurde die traditionsreiche Schwabinger Buchhandlung Lehmkuhl übernommen, und 2010 ging das lieferbare Bücherprogramm des Langewiesche Brandt Verlags in Ebenhausen an C.H.Beck über. Zu der seit den 
neunziger Jahren expandierenden Unternehmensgruppe zählt ferner eine große Kette juristischer Fachbuchhandlungen.

Die Zahl der festangestellten Mitarbeiter im Gesamtverlag stieg von 178 im Jahr 1970 über 447 im Jahr 2000 auf $5^{21}$ im Jahr 2012. ${ }^{11}$ Gleichzeitig sank der Anteil an männlichen Mitarbeitern von 4o Prozent auf 34, Prozent und die Zahl der Teilzeitbeschäftigten erhöhte sich von 15 auf 117 : Der Verlag spiegelt die Veränderungen des deutschen Arbeitsmarkts.

Schauen wir uns die Produktion des kulturwissenschaftlichen Verlagsteils an, der seit 1978 in einem großen, hellen Neubau an der Ainmillerstraße 12 residiert, den der Architekt Alexander von Branca entworfen hat. ${ }^{12}$ Das Grundstück hatte Heinrich Beck bereits 1954 erworben. ${ }^{13}$ Von 1974, über 1990 bis 2010 stieg die Anzahl der jährlichen Neuerscheinungen, Neuauflagen und Nachdrucke von 122 (darunter 81 Neuerscheinungen) über 189 (126) auf 395 (212) und die jährliche Buchproduktion von 411 527 (223737) über 1220470 (625870) auf 1851 514 (1 125985) Exemplare. Es zeigt sich ein deutlicher Trend, dass sowohl die Zahl der Neuerscheinungen als auch die der Nachdrucke und Neuauflagen stetig zunimmt. In jüngerer Zeit liegt die Anzahl der Neuauflagen und Nachdrucke nur zwischen zehn bis zwanzig Prozent unter derjenigen der Neuerscheinungen. Zum Vergleich: Im juristischen Bereich (ohne Vahlen) verdoppelte sich die Zahl der Neuerscheinungen zwischen 1990 (235) und 2010 (471), im selben Zeitraum erhöhte sich auch die Zahl der Neubearbeitungen und Nachdrucke von 153 auf 34,5. Die Produktionszahlen stiegen von 3 4,17 140 (darunter 2544370 Neuerscheinungen) auf 4320500 (3 104,430). Allerdings expandiert im juristischen Zweig das Online-Angebot seit dem Jahr 2001 rasant. Das höchst erfolgreiche Geschäftsmodell schwächt notwendigerweise den Printverkauf und verlangsamt das Produktionswachstum.

Die deutsche Verlagslandschaft ist seit den 1970er Jahren gekennzeichnet durch Übernahmen, Verflechtungen, Mehrheitsbeteiligungen und Konzentrationsbewegungen. ${ }^{14}$ C.H.Beck blieb ein unabhängiges Familienunternehmen. Seit dem Jahr 2000 firmiert es allerdings nicht länger unter «C.H. Beck'sche Verlagsbuchhandlung», sondern als «Verlag C.H.Beck». Doch die beiden Verlagsteile, der juristische und der kulturwissenschaftliche oder, wie sie sich ebenfalls seit dem Jahr 2000 nennen: «Recht - Steuern - Wirtschaft» und «Literatur - Sachbuch - Wissenschaft», haben sich auseinanderentwickelt. Schon der Internetauftritt von C.H.Beck erweckt den Eindruck, dass nicht ein Gesamtverlag, sondern zwei sehr unterschiedliche Verlage unter einem Dach existieren. ${ }^{15}$ Der juristische Zweig muss als Fachverlag für sein riesiges Wissensgebiet «Recht - Steuern - Wirt- 
schaft» das elektronische Angebot weiter ausbauen, um jedem Kunden ein quasi maßgeschneidertes und stets aktuelles Modul anbieten zu können zum Arbeitsrecht, zum Steuerrecht, zum Handelsrecht usw. Für den kulturwissenschaftlichen Publikumsverlag ist dies kein Modell. Er wendet sich an eine breite intellektuelle Öffentlichkeit und muss sich anderer vertrieblicher Mittel bedienen.

Die beiden Verleger, Hans Dieter und Wolfgang Beck, repräsentieren sehr unterschiedliche Denk- und Arbeitsweisen und letztlich zwei verschiedene Verlagskulturen. Es ist ein offenes Geheimnis, dass die Brüder kein allzu enges Verhältnis miteinander pflegen. Ein Bonmot - se non ̀̀ vero, è ben trovato -, das unter den Mitarbeitern des Hauses kursiert, spiegelt die Situation recht treffend. Auf einer Betriebsversammlung soll einer der Verleger gesagt haben: «Ich weiß nicht, welche Meinung mein Bruder in dieser Sache vertritt, aber ich bin gegenteiliger Meinung.» Kompromisse sind bisweilen schwer zu finden. Belastet war auch das 225. Verlagsjubiläum von 1988. Nicht nur über Einladungen bestand Dissens, sondern auch über die Darstellung der Verlagsgeschichte. Trotz aller manifesten Unterschiede zwischen Hans Dieter und Wolfgang Beck gibt es sehr wohl auch Gemeinsamkeiten. Beide sind Großverleger, die Einfluss auf die Entwicklung der von ihnen repräsentierten Gebiete nehmen; sie sind, um einen Begriff des Publizisten Heribert Prantl aufzugreifen, «Landschaftsgärtner» sowohl der Rechtswissenschaft wie der Kulturwissenschaft. ${ }^{16}$ Es gibt zudem gewisse habituelle Ähnlichkeiten. Ihr Handeln ist an der sozialmoralischen Norm der Sparsamkeit und Bescheidenheit orientiert, sie verachten Luxus und Verschwendung und legen eher eine asketische Haltung an den Tag. Ernst Augustin hat Wolfgang Beck in trefflicher Manier charakterisiert. Er beschreibt in seiner Rede, die er auf der Feier zum 65. Geburtstag des Verlegers hielt, wie er zur Ainmillerstraße $12 \mathrm{kam}$, um ein erstes Gespräch zu führen. Er zögerte «vielleicht einen Moment auf den Stufen, vielleicht in Erwartung des blauen Jaguars oder doch zumindest einer bläulichen Münchner Variante». Dabei übersah er «völlig den Radfahrer, der soeben auf seinem Fahrrad in die Einfahrt einbog»: Wolfgang Beck. ${ }^{17}$ Einen blauen Jaguar fährt natürlich auch Hans Dieter Beck nicht. Einen blauen Jaguar fuhr Siegfried Unseld.

Es griffe allerdings zu kurz, die Ursache des Auseinanderdriftens der Verlagsteile zu personalisieren. C.H.Beck ist aus betriebswirtschaftlichen Gründen notwendigerweise auf die Bedürfnisse des juristischen Bereichs ausgerichtet. Dass die divergierenden Interessen nicht zu noch tieferen Rissen geführt haben, ist nicht zuletzt den Mitarbeitern zu verdanken, die 


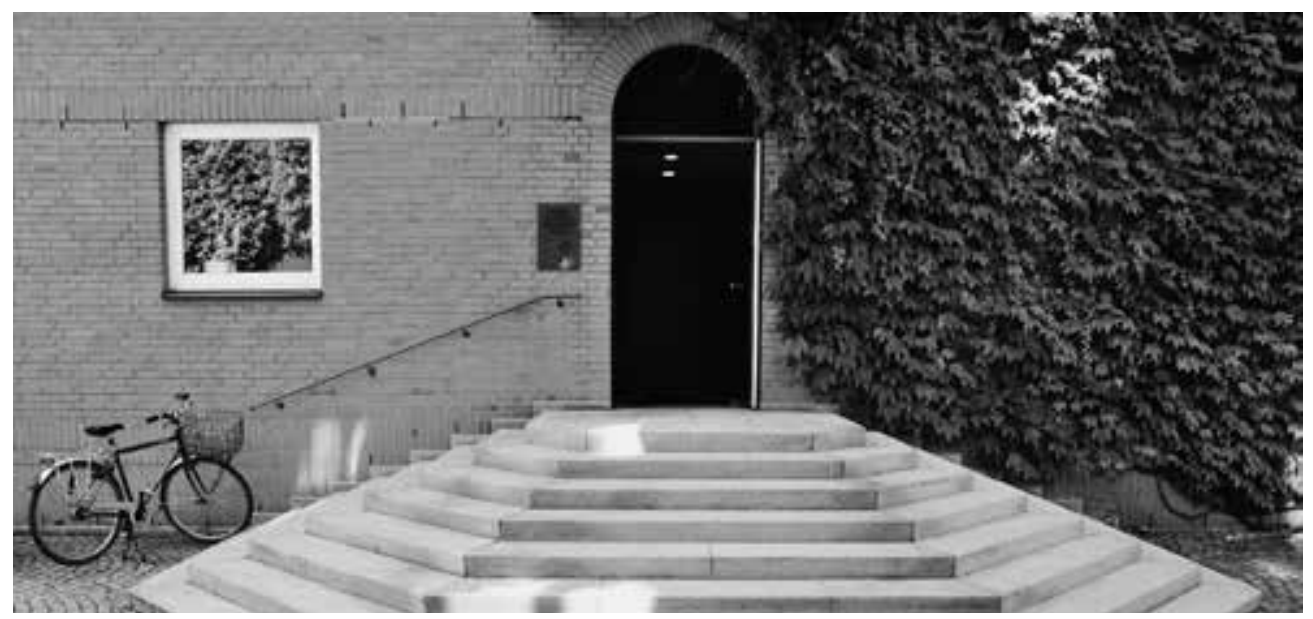

Fahrrad des Verlegers vor dem Verlagsgebäude in der Ainmillerstraße 12

über die Grenzen der Verlagszweige hinweg Kommunikation und Kooperation pflegen. Der Gesellschaftervertrag der Brüder hält das Unternehmen juristisch zusammen; und die lange Familientradition gibt vor, das Unternehmen in die Hände der siebten Generation zu legen. Aus der Ehe von Hans Dieter und Helga Beck sind drei Töchter hervorgegangen, Anja, geboren am 13. Januar 1988, Veronika, geboren am zo. August 1989, und Eva, geboren am 21. November 1991. Wolfgang Beck ist zum zweiten Mal verheiratet. Seiner ersten Ehe mit Juliane Beck entstammen die beiden Söhne Jonathan, geboren am 21. April 1977, und Valentin, geboren am 7. April 1979. Mit seiner zweiten Ehefrau Mahrokh Beck hat er eine Tochter, Nigin, geboren am 9. Februar 1984, und einen Sohn, Alexander, geboren am 13. Mai 1987. Mittlerweile ist Wolfgang Beck auch zweifacher Großvater, so dass, wenn alles gutgeht, auch eine achte Verlegergeneration heranwachsen und den langen Weg ins 22. Jahrhundert beschreiten könnte.

Während Wolfgang Beck sich nach dem Verlagsjubiläum 2013 allmählich aus dem Geschäft zurückziehen will, denkt Hans Dieter Beck noch nicht ans Aufhören. Als Nachfolger Wolfgang Becks steht sein Sohn Jonathan bereit. Der promovierte Volkswirt arbeitet seit 2008 im Hause und leitet zur Zeit das wirtschaftswissenschaftliche Programm des Vahlen-Verlags im Unternehmensbereich von Hans Dieter Beck. Auch über die Zukunft des Gesamtunternehmens hat sich Wolfgang Beck Gedanken gemacht: Er wünschte sich, dass den künftigen Gesellschaftern, sieben an der Zahl, ein Aufsichtsrat an die Seite gestellt würde, der künftig helfen könnte, die 


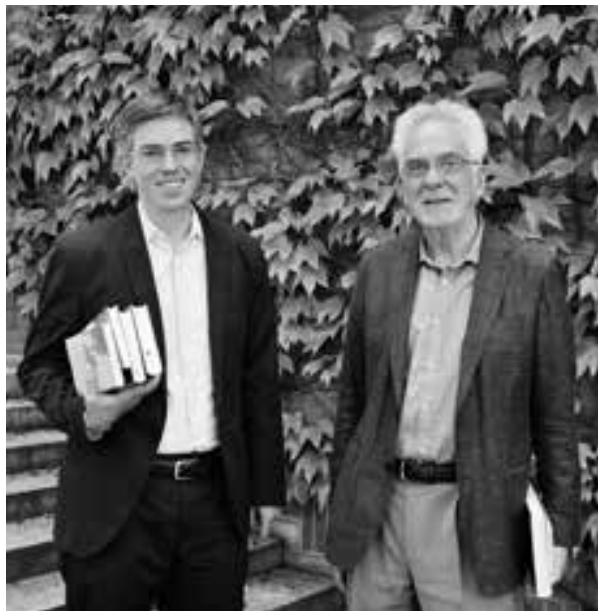

Jonathan und Wolfgang Beck

Unternehmensstrategie auszuarbeiten, und sich im Konfliktfall um Konsensfindung bemühte.

Sicher wird Wolfgang Beck auch dann, wenn er sein Büro geräumt hat, weiter als Förderer und Mäzen wirken. Für seine Verdienste um die Kulturwissenschaften im Allgemeinen und die Altertumswissenschaften im Besonderen hat er 2005 von der Philosophischen Fakultät der Universität Freiburg die Ehrendoktorwürde erhalten. Mitarbeitern, Autoren und Beratern gegenüber zeigt sich der Verleger grundsätzlich großzügig. Als die «tätige Cooperation» zwischen Walther Killy und dem Hause Beck, die in den sechziger und siebziger Jahren so erfolgreich gewesen war, 1990 nach 25 Jahren auf Wunsch des Verlegers endete, richtete er für Killy ein Abendessen in seinem Privathaus in der Klementinenstraße aus, zu dem ein handverlesener Kreis von Weggefährten eingeladen wurde. ${ }^{18}$

In welcher Weise der Verleger seine kulturelle Verantwortung wahrnimmt, zeigen anschaulich einige Projekte, die Wolfgang Beck als Verleger oder durch die C.H.Beck Stiftung gefördert hat oder noch immer fördert. ${ }^{19}$ Die Stiftung, die er gemeinsam mit seinem Bruder 2006 gegründet hat, stellt im Durchschnitt jährlich eine mittlere sechsstellige Summe für humanitäre sowie wissenschaftlich-kulturelle Vorhaben zur Verfügung. Zwischen 1993 und 1995 unterstützte der Verlag die Gründung des Münchner Literaturhauses mit 100000 Euro; hinzu kamen zwischen 2006 und 2010 mehrere Einzelspenden. Der C.H.Beck-Übersetzerpreis, der seit 1999 alle zwei Jahre vergeben wird, ist mit einem Preisgeld von 1000 ouro dotiert; auch die Kosten für die Jury trägt der Verlag. C.H.Beck-Stipendien am Deutschen Literaturarchiv in Marbach werden seit 2008 mit jährlich 15000 Euro finanziert. Den Lectures- und Visitorship-Programmen der American Academy wurde ebenso Förderung zuteil wie Stipendienprogrammen an der Ruperto Carola und der Hochschule für Jüdische Studien in Heidelberg. Das Historische Kolleg in München erhielt ein Förderstipendium, und das International Fellowship Program der Jerusalemer Buchmesse profitierte von finanziellen Zuwendungen. Wolfgang Beck engagierte und engagiert sich überdies für das Jüdische Museum in Frankfurt, für ungarische Übersetzungen wichtiger philosophischer Werke und 
für eine geplante vierbändige Anthologie zur spanischen und lateinamerikanischen Lyrik. Immer wieder hat der Verleger Druckkostenzuschüsse für Bücher gewährt, die nicht bei C.H.Beck erschienen. Sint Maecenates, non deerunt Marones. ${ }^{20}$

\section{«Corporate Identity»}

C.H.Beck besitzt heute eine erfolgreiche Unternehmenskultur, an der sowohl seine Autoren als auch seine Mitarbeiter partizipieren. Die Beschäftigten des Verlags werden übertariflich bezahlt. Die Spannungen, die sich 2010/11 durch die Eingruppierung in Tarifgruppen zeitweilig ergaben, ${ }^{21}$ sind inzwischen überwunden. Zu dem tariflich vereinbarten 1z. effektiven Monatsgehalt, dem sogenannten Weihnachtsgeld, kommt ein weiteres halbes Gehalt als Urlaubsgeld hinzu. Bei der Verabschiedung eines Mitarbeiters oder einer Mitarbeiterin in den Ruhestand erhält er oder sie ein Monatsgehalt als «Treueprämie», die allerdings nicht ausgezahlt wird, wenn er oder sie in Altersteilzeit geht. Alle Pensionäre werden, sofern sie noch in München und Umgebung leben, zu den Verlagsfesten eingeladen. Alle erhalten auch nach ihrer Pensionierung zu runden Geburtstagen ein Geldgeschenk und je nach ihrer Position auch ein persönliches Geschenk von den Verlegern. Jede Mitarbeiterin und jeder Mitarbeiter hat ferner nach zehnjähriger Zugehörigkeit zum Unternehmen einen unverfallbaren Anspruch auf eine monatliche finanzielle Zuwendung bei Erreichen des Rentenalters im Rahmen der betrieblichen Altersvorsorge.

Doch nicht nur übertarifliche Gehälter kommen den Angestellten zugute. Es hat zwar einige Jahre gedauert, bis die Verlagsleitung und der Betriebsrat sich auf die Einrichtung eines Betriebskindergartens verständigt hatten, doch nun unterstützt der Verlag seit 1996 eine solche Institution in der Ainmillerstraße. Die sogenannten Bücherwürmchen können dauerhaft kostenlos Räume im Nachbargebäude des Verlags nutzen, das den Verlegern gehört. Die laufenden Kosten des Kindergartens, der maximal 16 Kinder aufnehmen kann, werden durch Beiträge der Eltern (45 Prozent), Zuschüsse der Stadt München (ebenfalls 45 Prozent) und zusätzliche Spenden des Verlags ( 10 Prozent) gedeckt.

Für die Verleger versteht es sich von selbst, Mitarbeitern zu runden Geburtstagen, Hochzeiten oder Geburten zu gratulieren und auf diese Weise ihre Wertschätzung zu kommunizieren. Konstruktive Lösungen in umstrittenen Fragen, die der Unternehmensführung und der Belegschaft 
nützen, können meist einvernehmlich gefunden und umgesetzt werden. So stimmten Betriebsrat und Belegschaft der Regelung zu, dass nach Einführung der 37,5-Stunden-Woche zunächst weiterhin 38,5 Stunden pro Woche gearbeitet wurde. Die Mehrstunden wurden durch kollektive freie Tage ausgeglichen: Am Freitag zwischen Himmelfahrt bzw. Fronleichnam und dem darauffolgenden Wochenende sowie zwischen Weihnachten und Neujahr und am Faschingsdienstag blieb der Verlag geschlossen, ohne dass dafür Urlaub genommen werden musste. Die über weite Strecken spannungsfreie Entwicklung kennzeichnet indes nur den Münchner Verlag; in der Nördlinger Druckerei des Gesamtunternehmens kam es immer wieder zu Konflikten, die sich meist an der Wochenarbeitszeit und der Lohnentwicklung entzündeten. Die gewerkschaftlich organisierten Drucker scheuten, wenn sie ihre Rechte beschnitten oder sich unter Druck gesetzt fühlten, auch vor einem Arbeitskampf nicht zurück. Streiks beeinträchtigten bisweilen sogar die Verlagsproduktion. ${ }^{22}$

Zur Unternehmenskultur des Verlags C.H.Beck gehören bestimmte Veranstaltungen und Institutionen, Feste und Feiern, die die heterogene Belegschaft der beiden Verlagsteile integrieren. Folgen wir dem Lauf eines Jahres im Verlag. ${ }^{23}$ Es gibt, wie schon unter Heinrich Beck, jedes Jahr eine Weihnachtsfeier. In früheren Zeiten fand sie in der Regel in den Räumen des Verlagshauses statt. Jedes Jahr wird neben dem «Weihnachtsgeld» ein Bücherfreibetrag gewährt: Jeder Mitarbeiter darf sich im Wert von zur Zeit 150 Euro (Ladenpreis) Bücher aus dem Verlagsprogramm aussuchen. Darüber hinaus liegen Weihnachtspräsente auf dem Gabentisch: drei Flaschen Wein für die männlichen, individuelle Geschenke für die weiblichen Angestellten. Die Weine werden zuvor eigens von einem «Wein-Gremium» verkostet und ausgewählt. Die Geschenke für die Mitarbeiterinnen: Kosmetika, Lederwaren, Haushaltsartikel, Handtücher, Schmuck, Kunsthandwerk und CDs bis zu einem Wert von aktuell 35 Euro wurden lange von einem kleinen Team eingekauft, das die Geschenke grundsätzlich nicht in irgendeinem Kaufhaus einer Kette besorgte, sondern gezielt bei Händlern in der Schwabinger Nachbarschaft aussuchte. Die Präsente wurden auf einem Basar arrangiert, wo sich die Mitarbeiterinnen jeweils ihr Geschenk aussuchen durften. Seit 2009 ist die Anzahl der verschiedenen Präsente deutlich reduziert, um das Verfahren zu vereinfachen.

Im Jahr 1992 wurde erstmals der Vorschlag aus der Belegschaft umgesetzt, statt der Geschenke bedürftigen Menschen eine Spende zukommen zu lassen. Damals floss das auf diese Weise gesammelte Geld einem guten Zweck in Russland zu. Zwei Jahre später veranstaltete der Betriebsrat eine 
Umfrage unter der Belegschaft, nach der sich eine Mehrheit dafür aussprach, in jedem zweiten Jahr auf die Geschenke zu verzichten und den Gegenwert stattdessen zu spenden. Das wird seit 1995 im Haus so gehandhabt. 2002 wurde nach der Hochwasser-Katastrophe in Sachsen erneut per Umfragevotum entschieden, auch in diesem Jahr außerhalb des Turnus zugunsten einer Spende auf Präsente zu verzichten. Über die Spendenempfänger berät ein kleines Team, das aus den beiden Assistentinnen der Verleger, dem Betriebsratsvorsitzenden und einer Mitarbeiterin der Finanzabteilung besteht. Vorschläge aus der Belegschaft sind immer willkommen. Die Verleger haben die eingebrachten Vorschläge stets akzeptiert und die zu spendende Summe, meist etwa 20000 Euro, in manchen Jahren sogar noch auf zo ooo Euro erhöht. Die Empfängerliste der Spenden wird dann jeweils im Intranet und über Schwarze Bretter der gesamten Belegschaft mitgeteilt.

Nicht nur zu Weihnachten erhalten die Mitarbeiter Vergünstigungen. Wiederum setzen Wolfgang und Hans Dieter Beck damit die Gepflogenheiten des Vaters fort. Am Nikolaustag trifft man sich zum vorweihnachtlichen Kaffee mit Christstollen oder Lebkuchen. Zu Ostern bekommt jeder Mitarbeiter nach wie vor eine Tüte mit Schokoladen-Ostereiern. Zum 1. Mai erhielt jeder Mitarbeiter zusammen mit seiner Gehaltsabrechnung einen «Maitaler» in Form von fünf Mark, die in Münzen aufgeklebt waren. Nach Einführung des Euro wurde ein 5-EuroSchein verteilt. 2006 schenkte der Verlag seinen Mitarbeitern einmalig die Sonderprägung einer 10-Euro-Münze. Damit jedoch wurde diese Tradition abgeschafft, nicht weil man die Summe einsparen wollte, sondern weil der manuelle Aufwand bei der Verteilung zu groß geworden war.

Alle Mitarbeiter erhalten einen steuerfreien Zuschuss zum Mittagessen in Form von Essensmarken in Höhe von 42 Euro pro Monat (2,80 Euro pro Arbeitstag). Auf die Einrichtung einer Kantine verzichtete die Belegschaft. Statt Semmeln aus dem Automaten bevorzugte die große Mehrheit die Möglichkeit, an diesem - aufs Jahr gerechnet - nicht ganz unerheblichen zusätzlichen Einkommen festzuhalten, das den

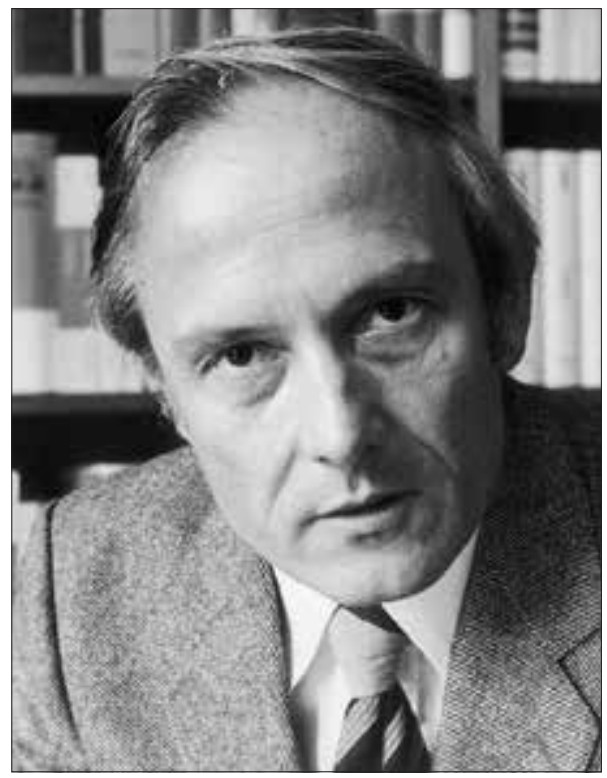

Hans Dieter Beck 
Verlag etwa im Jahr 2010 insgesamt 290000 Euro kostete. Die Marken, vulgo «Becktaler», werden in verschiedenen Lokalen und auch in einigen Supermärkten in Verlagsnähe angenommen.

Heinrich Beck schätzte die Betriebsausflüge. Auch nach seiner Zeit wurde fast jeden Sommer ein ganztägiger Ausflug mit der gesamten Belegschaft ins bayerische Umland unternommen. zoo Leute saßen in mehreren Bussen, die verschiedene Ziele ansteuerten, wo ein abwechslungsreiches Programm geboten wurde. 2000 wurde der Betriebsausflug als nicht mehr zeitgemäß aufgegeben: Stattdessen feiert man alljährlich ein Sommerfest in München, seit 2002 in einem Zelt im Verlagsgarten.

Autoren, dem nach einhelligem Credo wichtigsten Kapital des Verlags, werden andere Formen der Zuwendung zuteil. Der Erfolg der Autorenpflege beruht vor allem auf dem persönlichen Kontakt und der professionellen Betreuung. Natürlich bleibt eine sorgfältige Redaktion des Manuskripts auf dem Weg zum Buch die beste Empfehlung für einen Verlag. Aber es zeichnet das Haus aus, dass man sich für seine Autoren eben nicht nur dann interessiert, wenn ein neues Publikationsvorhaben ansteht. So verstehen sich Weihnachtskarten und Weihnachtsgeschenke bei C.H.Beck von selbst. Im kulturwissenschaftlichen Zweig wird seit Anfang der 199oer Jahre häufig die sogenannte Jahresgabe verschickt, wie dies auch manche andere Verlage tun. Dabei handelt es sich um ein schmales, jedoch herstellerisch kostbar gestaltetes Buch, das erst im darauffolgenden Frühjahr auf den Markt kommt. Aber auch andere Verlagswerke werden zu Weihnachten verschenkt. Sie werden zuvor in Geschenkpapier eingewickelt und mit einer persönlich unterschriebenen Weihnachtskarte versehen. Das Motiv lässt sich Wolfgang Beck selbst angelegen sein. Wenn ein Autor von mehreren Lektoren betreut wird, wird auch darauf geachtet, dass die Karten dann von allen Beteiligten unterschrieben werden. Rund 2000 Autoren des kulturwissenschaftlichen Verlagsteils werden auf diese Weise bedacht. Hier wird ein beachtlicher organisatorischer und materieller Aufwand betrieben, der die Bindung der Autoren an das Haus stärken soll.

Zur Autorenpflege gehört ferner, dass den Autoren auch zu runden Geburtstagen gratuliert und ein Präsent dargeboten wird. Wiederum geht es nicht um den materiellen Wert des Geschenks, sondern um die Stärkung der persönlichen Beziehung zwischen Autor und Verlag. Wenn ein mehrbändiges oder bedeutendes Werk abgeschlossen wird, lädt der Verleger durchaus auch schon einmal persönlich zu einem Essen ein; nicht selten steht dieses Event in Verbindung mit einer Buchvorstellung. Auf der Frankfurter Buchmesse bittet Wolfgang Beck seit 1978 zum Empfang in den Hes- 
sischen Hof. Zu dem Vortrag und zu dem anschließenden Empfang werden alle gebeten, die im kulturellen und wissenschaftlichen Leben Rang und Namen haben. Es fing mit etwa 80 Besuchern an, heute sind es regelmäßig mehrere Hundert. So versammelt der Verlag «eine Gelehrtenrepublik als Bürgergesellschaft, in der Engagement und Bildung sich gegenseitig befeuern». ${ }^{24}$ Sie alle sind gerne Autoren von C.H.Beck, weil sie sich persönlicher Wertschätzung sicher sind. Dies ist wahrlich keine Selbstverständlichkeit mehr in der Verlagswelt des 21 . Jahrhunderts. 
https://doi.org/10.17104/9783406654015-657, am 26.04.2023, 13:37:28

Open Access - (c) EY EY - http://www.beck-elibrary.de/agb 